\title{
Effect of Time, Solvent-Solid Ratio, Ethanol Concentration and Temperature on Extraction Yield of Phenolic Compounds From Olive Leaves
}

\author{
Noureddine Elboughdiri \\ Chemical Engineering Department \\ College of Engineering \\ University of Hail \\ Saudi Arabia and \\ Chemical Engineering Process Department \\ National School of Engineering Gabes \\ University of Gabes \\ Tunisia \\ ghilaninouri@yahoo.fr
}

\begin{abstract}
This study aims to determine the factors affecting the process of extraction of phenolic compounds from olive leaves. Two methods of extraction were used in this work and different tests were implemented with the aim of optimizing the recovery of phenolic compounds from olive leaves. The factors considered were the extraction time, temperature, solvent-solid ratio and the ethanol concentration. Obtained results show that the recommended optimal conditions for the extraction of the total phenolic compounds from olive leaves were found at $40^{\circ} \mathrm{C}$ with a solvent/solid ratio of $30: 1$ and ethanol concentration of $80 \%(v / v)$ when the dried olive leaves are stored at $-21^{\circ} \mathrm{C}$ at the beginning of the experiments.
\end{abstract}

Keywords-solvent extraction; total phenolics; olive leave; optimization

\section{INTRODUCTION}

The olive leaves have been used in traditional medicine in some Mediterranean regions. Many recent studies indicate that olive leaves contain significant amounts of phenols possessing strong and high antioxidant capacity [1], whose concentration are near to those of olive oil. Utilizations of olive leaves to produce health promoting products and food ingredients have great potential for pharmaceutical and food industries. There are many methods for the extraction of antioxidants from olive leaves, and solvent extraction is the most common one. The solvents frequently used for the extraction of phenolic compounds and derivatives from olive leaves are methanol or hexane and water mixtures. However, the toxicity of both solvents limits their industrial exploitation in order to obtain antioxidants for human use [2]. In this paper, it is aimed to define the optimum extraction conditions for phenolic compounds from dried olive leaves which allows the evaluation of different process variables such as solvent composition, temperature, time and solvent to solid ratio effects on total phenolic compound content in the extract.

\section{LITERATURE REVIEW}

The extraction yield is strongly influenced by the solvent, due to the different polarity and different antioxidant potential of compounds extracted [3]. Therefore, organic solvent of higher polarity is more effective in quantitative recovery for phenolic compounds. Non-polar solvent and methanol were reported in many studies as a good solvent for extraction of phenolic compounds from the plants including olive leaves. However, it may lead to unacceptable levels of toxic residues in the final extracts. Ethanol and water are most widely employed as solvents for safety and abundances reasons [3]. Authors in [4] revealed that pure ethanol was not effective as a solvent for extraction of phenolic compounds from olive leaves, and water has important role in extraction process by increasing the diffusion of phenolic compounds through plant tissues. Changes in ethanol concentration modify the physical properties of the solvent such as density, dynamic viscosity, and dielectric constant. Solubility of compounds would also be modified by changes in the ethanol concentration, and this may influence the extraction of phenolics [5]. Authors in [6] used $40 \%, 50 \%$ and $60 \%$ ethanol $(\mathrm{v} / \mathrm{v})$ in their study to investigate the effect of ethanol concentration on total phenolics yield. Ethanol, methanol, ethyl acetate, boiling water, hexane, diethyl ether, chloroform and butanol were the main solvents used by researchers. Of these solvents, aqueous methanol or ethanol was most commonly used and the concentration of solvent varied between $40 \%$ and $80 \%(\mathrm{v} / \mathrm{v})$. Extraction with $80 \%$ methanol (v/v) was reported as the most effective method for olive leaves polyphenols [7]. Authors in [2] suggested $80 \%$ aqueous ethanol (v/v) was the optimum solvent for extraction of the targeted phenolics from olive leaf and it can be used as 
replacement of toxic solvents (methanol, diethyl ether, chloroform ) to obtain bioactive phenols for human use.

The temperature has impact on solubility, diffusion coefficient and the stability of phenolic compounds. An increase in temperature and a decrease of viscosity significantly increase the diffusion rate. However, high temperature may degrade the phenolics [8]. A range of extraction temperatures and time were employed by researchers in the extraction of phenolic compounds from olive leaves. Generally less extraction time is required with increasing temperature. When ethanol was used as a solvent, the extraction process took place either at room temperature or elevated temperatures of $40^{\circ} \mathrm{C}$ for 2 days under agitation where water bath was used to achieve required temperatures [9]. With boiling water, 8 to 29 minutes extraction time was reported [7]. This kind of extraction is purely a static process and easy to operate. Many studies indicate that the solvent to solid ratio is normally expressed as the ratio of the volume of solvent $\left(\mathrm{mm}^{3}\right)$ to the weight of extraction sample (gram) and considered an important factor in extraction of phenolic compounds from olive leaves. The solvent to solid ratio employed by the researchers for olive leaf extraction varied hugely from 4 to 100 , but a ratio between 10 and 50 was mostly reported in the literature [9-10].

\section{MATERIALS AND METHODS}

The olive leaves were collected from a region in north Tunisia in August 2017. After collection, the leaves were transferred to the laboratory, the twigs were removed from leaves, and the fresh leaves were washed with tap water. The clean leaves were air dried at $20^{\circ} \mathrm{C}$ for 5 hours before continuing drying at $30^{\circ} \mathrm{C}$ in an incubator for 2 days [11]. Before each extraction, the dried olive leaves were ground in a coffee grinder to become fine powder and stored $\mathrm{N}_{2}$ gas purged glass jars covered with aluminum foil at $0^{\circ} \mathrm{C}$ until the extraction. Analytical grade methanol and absolute ethanol was purchased from ThermoFisher Scientific. Distilled water was used for preparing a solution of ethanol/water $80: 20(\mathrm{v} / \mathrm{v})$, 70:30 (v/v), 60:40 (v/v) and 50:50 (v/v). The prepared extracts were used for total phenols determination. Colorimetric reaction was measured by UV spectrophotometry and the Folin Ciocalteau reagent was used as an oxidizing agent. Dried olive leave samples were stored at $-21^{\circ} \mathrm{C}$, in order to ensure the minimum change or variability between samples used for extraction experiments and to avoid degradation of phenolic compounds. Total phenolic contents of the controlled samples were monitored every 5 days.

\section{RESULTS AND DISCUSSION}

\section{A. Stability of Dried Olive Leaves}

Figure 1 shows the changes of the controlled sample values during the extraction experiments. We see that the total phenolic content remained constant. The result suggests that dried olive leaves are relatively stable when stored at $-21^{\circ} \mathrm{C}$. According to [12], there was no significant degradation of total phenolics during experimental period.

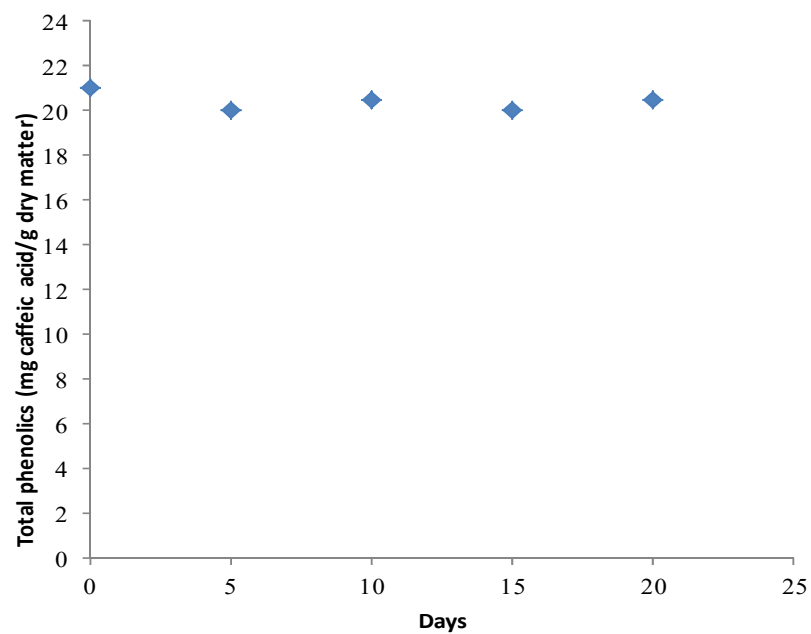

Fig. 1. Variation of total phenolic content of dried olive leaves with storage time at $-21^{\circ} \mathrm{C}$

\section{B. The Effect of Extraction Time on Extraction Yield}

Figure 2 shows the effect of extraction time on total phenolics content obtained in olive leaves extracts. Two extreme treatment conditions were tested in order to determine the optimum extraction time: the first treatment was carried out using $60 \%$ aqueous ethanol $(\mathrm{v} / \mathrm{v})$ at a solvent to solid ratio of $20(\mathrm{w} / \mathrm{w})$ and temperature of $20^{\circ} \mathrm{C}$, and the second treatment was using $80 \%$ aqueous ethanol $(\mathrm{v} / \mathrm{v})$ at a solvent/solid ratio of $30(\mathrm{w} / \mathrm{w})$ and temperature of $40^{\circ} \mathrm{C}$. Significantly higher level of total phenolic content was observed after 20 hours duration for both treatments. Comparing total phenolics obtained by the two treatments, it can be seen that significantly higher values of total phenolic compounds were found in the leave extract of the second treatment. It gives an indication that higher level of phenolic compounds may be recovered with increasing ethanol concentration, temperature and solvent to solid ratio.

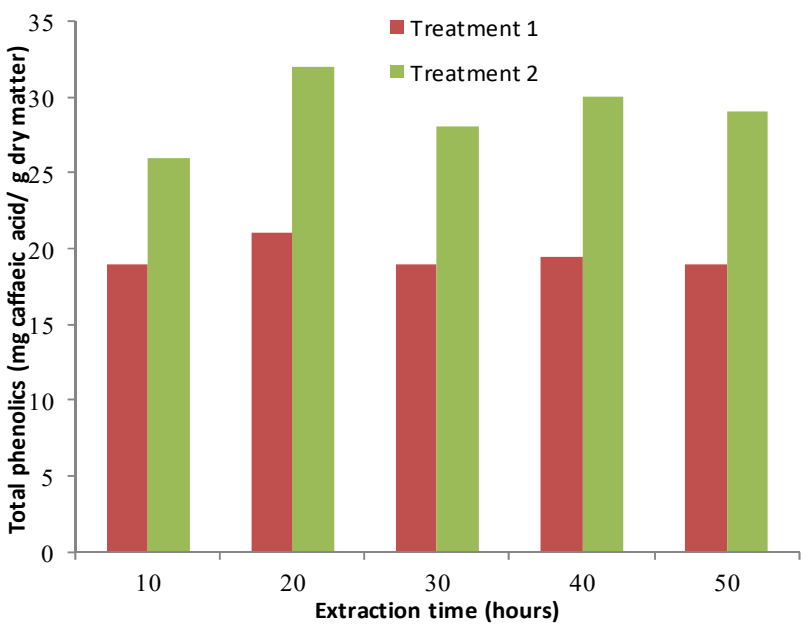

Fig. 2. Changes of total phenolic content in olive leaves extracts vs extraction time for two different treatments 


\section{The Effect of Extraction Temperature on Extraction Yield}

The impact of three extraction temperatures $\left(20^{\circ} \mathrm{C}, 30^{\circ} \mathrm{C}\right.$, $40^{\circ} \mathrm{C}$ ) on extraction yield was investigated. These temperatures were frequently used in previous studies [9-13]. Table I shows the effect of temperature on total phenolics extracted and indicates that the values were significantly affected by temperature. As can be seen in Table I, total phenolic compounds extracted at $40^{\circ} \mathrm{C}$ are significantly higher than those extracted at $20^{\circ} \mathrm{C}$ and $30^{\circ} \mathrm{C}$.

TABLE I. VARIATION OF TOTAL PHENOLICS VS. EXTRACTION TEMPERATURES

\begin{tabular}{|c|c|c|c|}
\hline Parameter & \multicolumn{3}{|c|}{ Value } \\
\hline Temperature $\left({ }^{\circ} \mathrm{C}\right)$ & 20 & 30 & 40 \\
\hline $\begin{array}{c}\text { Total phenolics (mg caffeic } \\
\text { acid/g dry matter) }\end{array}$ & 21 & 23 & 25 \\
\hline
\end{tabular}

Increasing temperature favored extraction by enhancing both the solubility and the diffusion coefficient from a solid matrix to a liquid matrix as mentioned earlier in the literature review [16]. As a consequence, much higher values of the total phenolics content are observed at $40^{\circ} \mathrm{C}$ than $20^{\circ} \mathrm{C}$. The results confirm that extraction temperature influences greatly the extraction yield. Increasing temperatures also affects the stability of phenolic compounds [17]. When extracting phenolic compounds from olive leaves at higher temperature, both effects need to be considered.

\section{The Effect of Solvent-Solid Ratio on Extraction Yield}

In this study, three different solvent-solid ratios $(20 \%, 25 \%$ and $30 \%$ ) were investigated. Result analysis for total phenolics indicates that total phenolics are significantly affected by solvent-solid ratio. The distribution of mean values of total phenolics for 5 extractions is shown in Table II. The higher the solvent to solid ratio $(30 \%)$, the higher the total phenolics obtained (24.5mg caffeic acid/g dry matter), suggesting that the solvent-solid ratio has a positive effect on the extraction yield. This is consistent with mass transfer principles. The driving force during mass transfer is the concentration gradient between the solid and the bulk of the liquid, which is greater, when, a higher solvent to solid ratio is used [5-14].

TABLE II. VARIATION OF TOTAL PHENOLICS VS. SOLVENT: SOLID RATIO

\begin{tabular}{|c|c|c|c|}
\hline Parameter & \multicolumn{3}{|c|}{ Value } \\
\hline Solvent: solid ratio (w/w) & 20 & 25 & 30 \\
\hline $\begin{array}{c}\text { Total phenolics (mg caffeic } \\
\text { acid/g dry matter) }\end{array}$ & 22 & 23.5 & 24.5 \\
\hline
\end{tabular}

\section{E. The Effect of Ethanol Concentration on Extraction Yield}

The influence of ethanol concentration on extraction yield was investigated. Ethanol concentrations of $50 \%, 60 \%, 70 \%$ and $80 \%(\mathrm{v} / \mathrm{v})$ were used in this study, which are the mostly reported concentrations in previous studies [9-15]. As shown in Figure 3, there was no significant difference in total phenolics obtained using ethanol at four different concentrations. Optimum ethanol concentration for olive leaves extraction reported in previously published studies varies. Authors in [6] used $40 \%, 50 \%$ and $60 \%$ ethanol $(\mathrm{v} / \mathrm{v})$ to extract phenolic compounds from olive leaves and they discovered that the ethanol concentration had a biphasic effect, as an intermediate ethanol level $(50 \%)$ appeared to provide the lowest yield. Higher concentration of total phenolics was obtained in extracts using $40 \%$ and $60 \%$ ethanol $(\mathrm{v} / \mathrm{v})$. It was suggested that decreasing or increasing the ethanol concentration both favored extraction of phenolics from olive leaves.

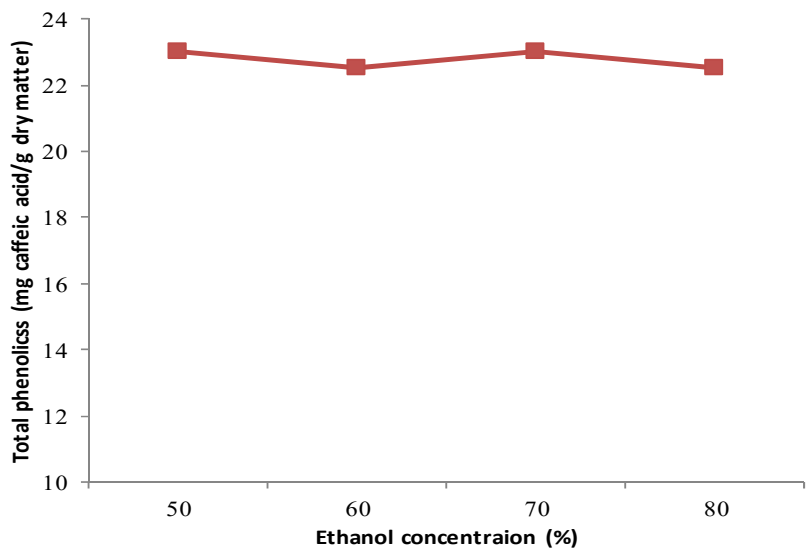

Fig. 3. Variation of total phenolics vs ethanol concentration

Authors in [18] suggested that $80 \%$ aqueous ethanol (v/v) was the optimum solvent for extraction of the targeted phenolics from olive leaves.

\section{CONCLUSIONS}

Olive leaves are a good and cheap source of phenolic compounds. The recovery of phenolic compounds from olive leaves could be achieved by solvent extraction. The solvent should be of low toxicity, economical and easily recycled by evaporation. In this study, the time, temperature, solvent to solid ratio and ethanol concentration were investigated to identify optimum extraction conditions. It was found that higher total phenolic content is obtained by increasing temperature and solvent to solid ratio. The results show that good recovery of phenolic compounds can be achieved using $80 \%$ ethanol $(\mathrm{v} / \mathrm{v})$ at $40^{\circ} \mathrm{C}$ and solvent to solid ratio of 30 . The olive leaf extract is recommended to be stored under $-21^{\circ} \mathrm{C}$. Three months storage at room temperature for liquid olive leaves extracts can lead to considerable loss of phenolic compounds, and thus prolonged storage should be avoided for liquid olive leaves extracts.

Olive leaves are a promising source for exploitation in terms of its total phenolic content. Current study results could be the first step for the implementation of the process on a large scale, being an adequate starting point for further studies regarding the optimization of the continuous process, of major interest from an industrial point of view.

\section{REFERENCES}

[1] M. H. Aludatt, I. Alli, K. Ereifej, M. N. Alhamad, A. Alsaad, T. Rababeh, "Optimisation and characterisation of various extraction 
conditions of phenolic compounds and antioxidant activity in olive seeds", Natural Product Research, Vol. 25, No. 9, pp. 876-889, 2011

[2] R. Japon-Lujan, J. M. Luque-Rodriguez, M. D. L. DeCastro, "Multivariate optimisation of the microwave-assisted extraction of oleuropein and related biophenols from olive leaves", Analytical and Bioanalytical Chemistry, Vol. 385, No. 4, pp. 753- 759, 2006

[3] E. B. Seda, "The effects of time, temperature, solvent:solid ratio and solvent composition on extraction of total phenolic compound from dried olive (olea europaea 1.) leaves", The Journal of Food, Vol. 35, No. 6, pp. 4-7, 2010

[4] E. Altiok, D. Baycin, O. Bayraktar, S. Ulku, "Isolation of polyphenols from the extracts of olive leaves (Olea europaea L.) by adsorption on silk fibroin", Separation and Purification Technology, Vol. 62, No. 2, pp. 342-348, 2008

[5] J. E. Cacace, G. Mazza, "Optimization of extraction of anthocyanins from black currants with aqueous ethanol", Journal of Food Science. Vol. 68, No. 1, pp. 240-248, 2003

[6] S. Mylonaki, E. Kiassos, D. P. Makris, P. Kefalas, "Optimisation of the extraction of olive (Olea europaea) leaf phenolics using water/ethanolbased solvent systems and response surface methodology", Analytical and Bioanalytical Chemistry, Vol. 392, No. 5, pp. 977-985, 2008

[7] N. S. A. Malik, J. M. Bradford, "Recovery and stability of oleuropein and other phenolic compounds during extraction and processing of olive (Olea europaea L.) leaves", Journal of Food Agriculture and Environment, Vol. 6, No. 2, pp. 8-13, 2008

[8] D. L. Luthria, "Influence of experimental conditions on the extraction of phenolic compounds from parsley (Petroselinum crispum) flakes using a pressurized liquid extractor", Food Chemistry, Vol. 107, No. 2, pp. 745752,2008

[9] K. Kiritsakis, M. G. Kontominas, C. Kontogiorgis, D. HadjipavlouLitina, A. Moustakas, A. Kiritsakis, "Composition and Antioxidant Activity of Olive Leaf Extracts from Greek Olive Cultivars", Journal of the American Oil Chemists Society, Vol. 87, No. 4, pp. 369-376, 2010

[10] A. P. Pereira, I. Ferreira, F. Marcelino, P. Valentao, P. B. Andrade, R. Seabra, J. A. Pereira, "Phenolic compounds and antimicrobial activity of olive (Olea europaea L. cv. Cobrancosa) leaves", Molecules, Vol. 12, No. 5, pp. 1153-1162, 2007

[11] O. H. Lee, B. Y. Lee, J. Lee, H. B. Lee, J. Y. Son, C. S. Park, Y. C. Kim, "Assessment of phenolics-enriched extract and fractions of olive leaves and their antioxidant activities", Bioresource Technology, Vol. 100, No. 23, pp. 6107-6113, 2009

[12] I. Mourtzinos, F. Salta, K. Yannakopoulou, A. Chiou, V. T. Karathanos, "Encapsulation of olive leaf extract in beta-cyclodextrin", Journal of Agricultural and Food Chemistry, Vol. 55, No. 20, pp. 8088-8094, 2007

[13] R. Japon-Lujan, P. Janeiro, M. D. L. DeCastro, "Solid-liquid transfer of biophenols from olive leaves for the enrichment of edible oils by a dynamic ultrasound-assisted approach", Journal of Agricultural and Food Chemistry, Vol. 56, No. 16, pp. 7231-7235, 2008

[14] M. Pinelo, M. Rubilar, M. Jerez, J. Sineiro, M. J. Nunez, "Effect of solvent, temperature, and solvent-to-solid ratio on the total phenolic content and antiradical activity of extracts from different components of grape pomace", Journal of Agricultural and Food Chemistry, Vol. 53, No. 6, pp. 2111-2117, 2005

[15] H. Jemai, M. Bouaziz, I. Fki, A. ElFeki, S. Sayadi, "Hypolipidimic and antioxidant activities of oleuropein and its hydrolysis derivative-rich extracts from Chemlali olive leaves", Chemico-Biological Interactions, Vol. 176, No. 2-3, pp. 88-98, 2008

[16] T. V. Gabbay Alves, R. Silva da Costa, B. Aliakbarian, A. A. Casazza, P. Perego, M. S. Pinheiro Arruda, J. O. Carréra Silva Júnior, A. Converti, R. M. Ribeiro Costa, "Bioactive compounds and antioxidant potential for polyphenol-rich cocoa extract obtained by agroindustrial residue", Natural product research, Vol. 11, No. 10, pp. 4-7, 2017

[17] X. U. Cong-Cong, B. Wang, P. U. Yi-Qiong, T. Jian-Sheng, T. Zhang, "Advances in extraction and analysis of phenolic compounds from plant materials", Chinese Journal of Natural Medicines, Vol. 15, No. 10, pp. 721-731, 2017
[18] R. Japon-Lujan, L. DeCastro, "Superheated liquid extraction of oleuropein and related biophenols from olive leaves", Journal of Chromatography A, Vol. 1136, No. 2, pp. 185- 191, 2006 